\title{
DESIGN OF A CNFET ARRAY FOR SENSING AND CONTROL IN P450 BASED Biochips For MUltiple DRUG DeteCtion
}

\author{
Shashikanth Bobba, Sandro Carrara and Giovanni De Micheli \\ Integrated Systems Lab (LSI), EPFL, Lausanne (Switzerland) \\ \{shashikanth.bobba, sandro.carrara, giovanni.demicheli\}@epfl.ch
}

\begin{abstract}
Bio-detection specially dedicated to distributed diagnostics is emerging as a quite important application for Nanobioelectronics. Bio-detection is required to be highly sensitive in order to succeed in sensing small amount of bio-markers in patient's blood sample. Carbon Nanotubes (CNTs) provides devices in the scale of the target molecules, thereby opening up possibilities to sense few bio-markers. Moreover, bio-detection is also required to be highly specific in order to succeed in disease discrimination. FET technology provides control in multi-panel biochip to enhance specificity. The aim of the present paper is to design an array of Carbon Nanotube Field Effect Transistors (CNFETs) to provide nano-biosensing based on cytochromes P450. In particular, a novel CNFET biosensor array design is proposed which is robust to the imperfections in the CNTs thereby achieving high level of sensitivity. The proposed CNFET based design assures the improved specificity by means of a multi-gate controller at the nano-scale. The proposed application in distributed diagnostics is on detection of drugs in multi-components samples by multiplexing different $P 450$ probes.
\end{abstract}

\section{INTRODUCTION}

$\mathrm{B}$ IONANOSENSORS, the integration of biological processes and molecules with nanoscale fabricated structures, offers the potential for electronic control and sensing of biological systems [1]. To achieve this goal, we envision the integration of fully functional biological systems and nanoelectronics. Among such prominent technologies that are being investigated, Carbon Nanotube (CNT) based biosensors are promising devices to enhance conventional biosensors in terms of sensitivity, scalability and ease in realizing complex massive sensor arrays. We propose a Carbon Nanotube Field Effect Transistors (CNFETs) based array for detecting multiple drugs to initiate point-of-care applications in personalized therapy.

Personalization of the therapy is highly desirable when compared to the state-of-the-art drug therapies. Only a fraction of patients, typically in the 20 to $50 \%$ range, receives benefit in major diseases [5]. This is because of the protein's polymorphism in metabolism [6] that causes over-dosing or under-dosing in hospitalized patients [7]. Therefore, any drug therapy needs to be personalized to the individual patient [8]. However, only monitoring systems for genetic predisposition to drug metabolism are available in the market [9] while the mean plasma concentration after a single drug dose varies on a patient-by-patient basis [10] even related to environmental conditions and patient's state. For these reasons, reliable point-of-care technologies to monitor multiple drug compounds in patient's plasma are absolutely required in therapy personalization. These technologies have to be offered at a low cost while keeping them simple to succeed in distributed diagnostics.

CNFET based bio sensors are very promising to fill the gap needed by the tools for personalized therapy.
Nevertheless, several challenges remain. First, it is desired to reliably fabricate devices which are robust to imperfections resulted from the state-of-the-art CNT synthesis technique [2, $3]$. This mainly requires CNFETs which are tolerant to the presence of metallic CNTs $[4,20]$ and which are robust to mispositioned CNTs $[2,3]$. Second, new strategies to integrate several specifically-targeted sensing-probes on CNFET array have to be developed. This integration addresses new design flow and tools required in personalized therapy.

The aim of the present paper is to address these open challenges both on imperfection immune systems and on bioprobes integration strategies. The rest of the paper is organized as follows. Section 2 deals with the detection principle; Section 3 gives a quick background on P450 multipanel sensors for bio-medical applications; Section 4 explains the CNFET synthesis to realize a robust CNFET-P450 sensor array; Section 5 describe a multiple drug detection employing eight-nodes CNFET-P450 sensor array; Section 6 summarize the paper conclusions.

\section{CNFET BASED SENSOR CONFIGURATION}

The representative CNFET-P450 sensor configuration is shown in Figure 1. Highly doped $(\mathrm{p}+)$ CNTs over the bulk form the channel between the source and drain contacts, where as the intrinsic CNT region under the gate is controlled by the gate voltage [21]. Turning-on the transistor while exposing the CNT-channel to a P450 solution leads to electrostatic drift [12] of P450 cytochromes towards the

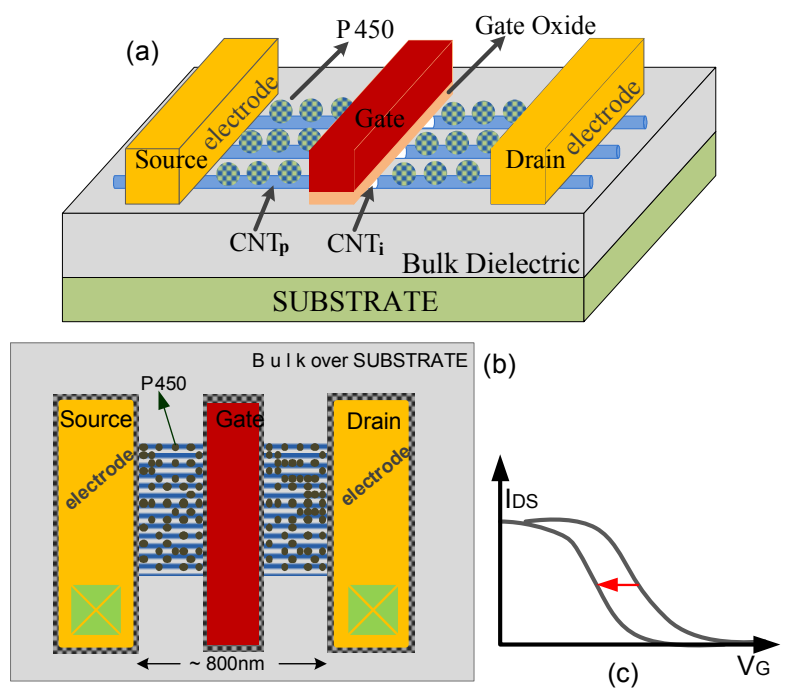

Figure 1. (a) Carbon Nanotube Field Effect Transistor with P450s bonded to the CNTs. (b) Top view of p-type CNFET 
nanotubes. The coupling between nanotubes and P450 cytochromes is assured by physical adsorption onto carbon nanotubes [14] or by chemical bonding [12]. As schematically shown in Figure 2, the presence of drug molecules which are targets of the $\mathrm{P} 450$ probe-proteins leads to charge transfer from the CNT channel to the protein. Similar charge transfer may be observed from proteins to the CNT by reversing the bias potential enabling the redox reaction on the drug.

Each protein needs two electrons in order to detect the single drug molecule. The $\mathrm{P} 450$ protein may receive these two electrons only if the CNT channel is operating by a proper gate voltage. As a consequence the charge transfers to the CNT shifts the DC towards more positive (electron donation from the drug to the CNT channel in the presence of target drug) or negative (hole donation from the drug to the CNT channel in the presence of the same drug but in reversed redox potential) gate voltages. Higher sensitivity can be achieved by maximizing the shift in the DC curve. This could be achieved by pushing the channel length to the maximum as observed in the case of $800 \mathrm{~nm}$-long CNT channel bonded to Streptavidin molecule to detect DNA duplex formation [19].

\section{BIO-PROBES For SPECIFIC DETECTION}

New devices to measure multiple drugs concentration in real-time may be based on multi-panel cytochromes P450 biochip which may detect multiple drugs directly in patient's serum. However, each P450 cytochrome detects many drugs and different P450s may detect the same drug. Therefore, a special design of CNFET array logic to control the accessibility of each and every protein-probe, in order to assure multiple queries to each P450 array spots [11], is proposed here. The proposed approach maintains high reliability and low cost of experimentation meanwhile it improves the specificity of Hybrid-CNFET biochip in drugs monitoring.

Cytochrome P450 (also abbreviated with CYP, or CYP450) is a super-family of heme-proteins found in Bacteria, Archaea and Eukaryotes [13]. Cytochromes P450 are involved in metabolism of a plethora of both exogenous and endogenous compounds. Most P450s metabolize multiple substrates, and many catalyze multiple reactions, which accounts for their central importance in metabolizing the potentially endless variety of endogenous and exogenous molecules. In drug metabolism, cytochrome $\mathrm{P} 450$ is probably the most important proteins of oxidative metabolism pathways in humans. In this context, the metabolism is any modification or degradation of chemicals including drugs and other endogenous compounds.
The whole cytochromes P450 family is capable of metabolizing a vast number and variety of organic substrates, estimated to be in the region of 200,000 chemicals or more, and involving around 60 distinct classes of biotransformation reactions. Metabolism mediated by cytochrome P450 enzymes plays a key role in the development of new drugs and in individual drug therapy. Thus, different P450 cytochromes may be considered to develop a multi-panel biochip for detection of multiple drugs content in human serum. Different $\mathrm{P} 450$ proteins detect different drugs. For example, the P450 isoform called 2B4 detects the benzphetamine (BZ), aminopyrine (AP) and 7-pentoxyresorufin (PX). The benzphetamine is an anoretic compound closely related to amphetamine. It is often used in anti-obesity therapy. The aminopyrine is a drug with analgesic, anti-inflammatory, and antipyretic properties. The 7-pentoxyresorufin is indicated for infections, hischemia, hypertrophy, obesity, etc. Another example is by the isoform P450 3A4 which may be used to detect cyclophosphamide (CP), dextromethorphane (DX) and midazolam (MZ). Cyclophosphamide is an anti-cancer agent; dextromethorphane is an analgesic, while midazolam is a benzodiazepine derivative with powerful anxiolytic, amnestic, hypnotic, anticonvulsant, skeletal muscle relaxant and sedative properties.

These two P450 isoforms are clear examples of proteins which may be used as probe enzymes in CNFET based biochip to obtain a multiple-drugs monitoring system. In the present paper, all the $\mathrm{P} 450$ proteins present in Table 1 are considered in order to propose an innovative design of a P450 based biochip for multiple-drugs detection based on CNFET logic. It is well know that the carbon nanotubes enhance the detection sensitivity in P450 based biosensors [11, 14, 16, 17]. Thus, functionalizing a CNFET by using the proper P450 isoform is used to obtain both an improvement in drugs detection and a control of each single probe thanks to the FET gate, as shown in Figure 3.

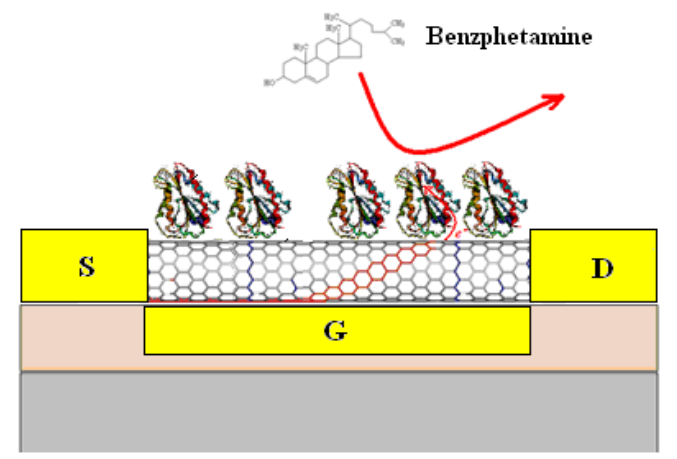

Figure 2 CNFET sensor functionalized by P450 2B4 to detect the benzphetamine, a powerful anoretic compound.

Table 1: Interaction map of the biochip P450 cytochromes and the target molecules considered in this study.

\begin{tabular}{|c|c|c|c|c|c|c|c|}
\hline P450-1A2 & P450-3A4 & P450-2B4 & P450-2B6 & P450-2C19 & P450-2C9 & P450-2D6 & P450-2E1 \\
\hline $\begin{array}{c}\text { Anti- } \\
\text { hypertensive }\end{array}$ & $\begin{array}{c}\text { Macrolide } \\
\text { antibiotics }\end{array}$ & $\begin{array}{c}\text { Anti- } \\
\text { inflammatories }\end{array}$ & $\begin{array}{c}\text { Anti-cancer } \\
\text { agents }\end{array}$ & $\begin{array}{c}\text { Anti- } \\
\text { epileptics }\end{array}$ & $\begin{array}{c}\text { Hypo-glycemic } \\
\text { agents }\end{array}$ & Beta blockers & Anesthetics \\
\hline- & $\begin{array}{c}\text { Anti- } \\
\text { arhythmics }\end{array}$ & Anti-obesity & & - & $\begin{array}{c}\text { Angiotensin-II } \\
\text { blockers }\end{array}$ & $\begin{array}{c}\text { Anti } \\
\text { depressants }\end{array}$ & - \\
\hline- & $\begin{array}{c}\text { HIV } \\
\text { Antivirals }\end{array}$ & - & & - & $\begin{array}{c}\text { Anti- } \\
\text { inflammatories }\end{array}$ & antipsychotics & -
\end{tabular}


(a)

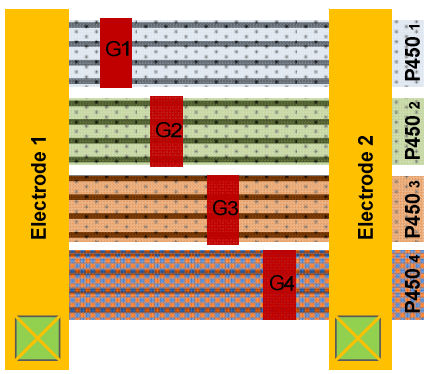

(b)

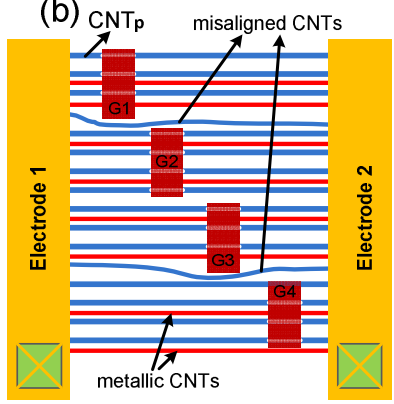

(c)

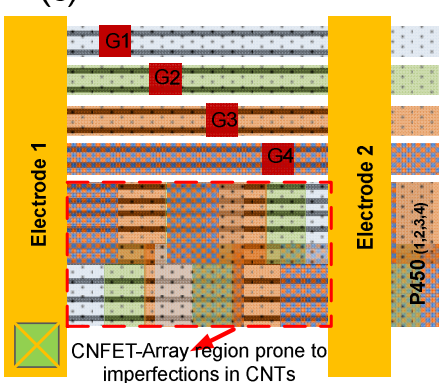

(d) Etched regions

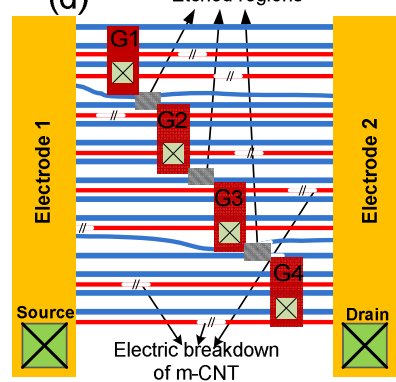

Figure 3. CNFET-P450 Sensor Array of index four (CSA4) (a) Ideal case (b) A p-type CNFET array prone to CNT imperfections (metallic -in red- and misaligned CNTs) (c) Effect of imperfections on the CSA (d) CSA immune to CNT defects.

\section{CNFET-P450 SENSOR ARRAY FOR MULTI-DRUG DETECTION}

\section{A. Ideal CNFET-P450 Sensor Array}

An ideal CNFET Sensor Array (CSA) for multi-drug detection comprises of multiple gates as shown in the Figure 3a. As a first step to build the CNFET array, semiconducting CNTs are grown or transferred to a substrate. The four gates (G1, G2, G3 and G4) and the contacts are then defined using lithography. Next, the CNTs are doped p-type with the gates masking the CNT segments under them thereby forming a CNFET Array (CA) having four control gates. The CNTs spanning under the four gates are distinct and hence the gates have independent control over the current flowing between the two electrodes. The one-to-one binding of P450 cytochromes and the gates is performed by activating each gate at a time while exposing the CNT channel to the respective P450 solution. During the exposure of a specific protein solution, respective P450 isoform is trapped onto the proper CNTs bundle driven by the single gate by using protein electrostatic trapping [12]. For example, applying an input code of 0111 $(\mathrm{G} 1=0 ; \mathrm{G} 2=\mathrm{G} 3=\mathrm{G} 4=1)$ to the CNFET array while exposing the array to solution containing $\mathrm{P} 450-1 \mathrm{~A} 2$ results in binding P450-1A2 to the doped-CNT segments spanning the gate G1. Iterating this step by activating one gate at a time will result in a CSA.

\section{B. Impact of CNT Imperfections on the Sensor Array}

Current CNT synthesis techniques are prone to many variations and imperfections in CNT parameters. The two major challenges that have a huge impact on the functionality and sensitivity of CSA are the presence of metallic CNTs and mispositioned CNTs. Current CNT synthesis techniques yield around 30\% metallic CNTs [15]. Although recent work has demonstrated growth/transfer of aligned CNTs [18], the probability of a few CNTs being mispositioned is nonnegligible. Figure $3 \mathrm{~b}$ shows a realistic scenario with considerable number of metallic CNTs and a few mispositioned CNTs. Mispositioned CNTs tend to be completely doped, as these CNTs are not located under the gates, thereby shorting the two electrodes. On the other hand, even metallic CNTs short circuit the two electrodes.
Quantifying the defective CNTs, i.e., sum of metallic and mispositioned CNTs, yields around $40-50 \%$ CNTs in the CNFET array which short circuit the two electrodes. Consequently, when the CA is subjected to different input codes to bind the four gates with respective $\mathrm{P} 450 \mathrm{~s}$, the defective CNTs bind all the four P450s (Figure 3c). This behavior leads to reduced specificity of the CSA on drug detection.

\section{Imperfection Immune CNFET Sensor Array}

The presence of misaligned and metallic CNTs lead to reduced sensitivity of the CSA towards the drug. Hence a CSA, robust to major imperfections is vital for realizing a reliable biochip. Etching out the CNTs at the crucial region provides immunity against misaligned CNTs [2]. Figure 3d demonstrates the introduction of etched regions on the CNFET array to nullify the effect of the two misaligned CNTs. This step does not need any additional lithography step prior to the formation of the array. The array comprises of the pCNFETs and the metallic-CNTs. Giving an input gate code of $1111(\mathrm{G} 1=\mathrm{G} 2=\mathrm{G} 3=\mathrm{G} 4=1)$ while applying high voltage between the two electrodes burn away all the metallic-CNTs. At this stage, we obtain a robust CNFET array. The binding of $\mathrm{P} 4501, \mathrm{P} 4502, \mathrm{P} 450_{3}$ and $\mathrm{P} 4504$ to the gates $(\mathrm{G} 1, \mathrm{G} 2, \mathrm{G} 3$ and G4) is done by activating each gate at a time $\{(\mathrm{t} 0,0111)$, ( $\mathrm{t} 1$, 1011), (t2, 1101), (t3, 1110)\} while exposing the CNT channel to the respective $\mathrm{P} 450$ solution.

\section{Multiple Drug Detection}

As listed in Table 1, different P450 isoforms have the same drug compound as substrate and different drugs are substrates of the same $\mathrm{P} 450$ protein. A possible array design to immobilize P450s for sensing multiple drugs has to consider redundancy and multiple enzyme-substrate interactions. A CSA with the eight different $\mathrm{P} 450$ isoforms presented in Table 1 is shown in Figure 4. Figure 4a is a direct physical extension of the CSA4 presented in the previous section. The main constraint while stacking several gates in between two electrodes is the distance between the two electrodes. The distance between two electrodes should be less than the average CNT length grown over a substrate. However, area efficient design can be obtained folding the layout as shown in the Figure $4 \mathrm{~b}$. On the other hand, the number of P450s bonded 
per gate is directly proportional to the area of the doped-CNT region, which is higher for the CSA8 in Figure $4 \mathrm{a}$ when compared to $4 \mathrm{~b}$. Moreover, the sensitivity of the CSA is proportional to the amount of $\mathrm{P} 450 \mathrm{~s}$ bonded to the conducting CNTs. Hence with the above presented analysis, an optimal number of gates stacked between the two electrodes could be analytically derived aiming towards optimizing area for a desired sensitivity level. In this case, we present CSA8 (CNFET Sensor Array of index eight) with which we can monitor multiple drugs. Moreover, the designed CSA8 enables dynamic spot query which may be used to improve

(a)
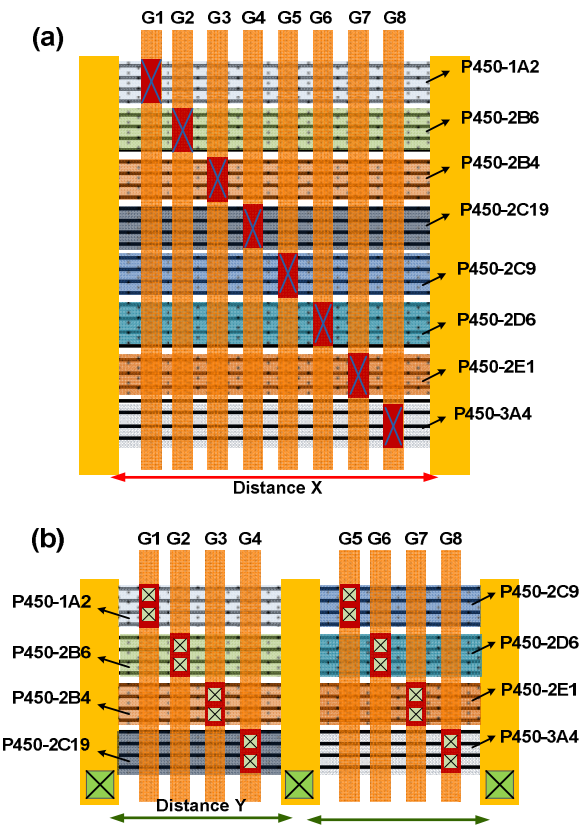

Figure 4. CNFET Sensor Array with eight probes (CSA8) (a) CSA8 with two electrodes. (b) CSA8 with three electrodes.

drugs specificity in case of multiple P450-drug interactions [11]. In case of samples having more drugs, different CNFET channels will give signals related to drugs detection. Independent query, activating a single channel, is realized by giving an appropriate input code to the gates of the array.

In such a manner, sub-sequent queries to the same channel are used to distinguish different drugs giving signals in different probes. For example, Table 1 shows that antiinflammatory compounds have an influence on both 2B4 and $2 \mathrm{C} 9$, hence a signal can be detected in G3 and G5. In case of signals from both these channels, a second query to the array by using slightly different polarity between the CNT-channel and the sample solution may enable us to distinguish between anti-inflammatory, anti-obesity and hypo-glycemics compounds. In case of $2 \mathrm{C} 9$ based detection, the ability to distinguish diclofenac, an anti-inflammatory compound, from the torsemide, a well known diuretic, is in $22 \mathrm{mV}$ in the current peak position [18]. Therefore, multiple query of differently polarized CNFET channel with different bias potentials improves the specificity in drugs detection. The simple design flow, mentioned above, enable us to solve the covering problem between the $\mathrm{P} 450$ cytochromes (probes) and the drugs (targets) and show how the presented CNFET sensor array platform can be employed to succeed on it. The optimum algorithm should sense the drugs with a minimum number of queries. The query list is generated by the firmware tools which accounts for the biochips design (the list of probes) and then sent to the sensor gates the input codes ( 8 bit in this example).

\section{CONCLUSION}

In the present paper, we investigated a multi-panel CNFET biochip design for detecting different drugs present in patients' sample. As a future work we plan to setup the design flow for simulating the configuration of the CNFET array with different P450s, eventually leading towards practical implementation of the sensor array. Such a sensor array enables early identification of drug candidates and it opens up new applications in personalization of drugs-therapy. The potential socio-economical relevance of a similar CNFET biochip technology is envisaged in a huge positive impact on different items: improvement of drugs efficacy, improvement of life quality of patients, diminishing of medical care expenses as well as shortening the time-to-market for new drug development. In fact, it may provide a unique and innovative solution for new tools in pharmacokinetic studies both in animals and humans. These new tools may open the possibility for shortening the typically 1-2 months now available with current measurement methods (e.g., LCMS/MS). The proposed CNFET sensor array inherently has the multiplexing nature as opposed to the sequential operation of the current methods. Therefore, an optimal use of such a multi-panel biochip may accelerate drug development by several months, and it will save costs for analytics by orders of magnitude.

\section{REFERENCES}

[1] Willner, I., et al., Science 2002, vol. 298, pp. 2407 - 2408

[2] Patil, N., et al., IEEE TCAD, pp. 1725-1736, 2008.

[3] Bobba, S., et al., IEEE Proc. DATE, pp. 616 - 621, 2009.

[4] Collins, P., et al., Science 2001, vol. 292, pp. 706 - 709, 2001.

[5] Lazarou, J., et al., JAMA. 279 (1998)1200-5

[6] Frank, D., et al., Eur J Clin Pharmacol (2007) 63:321-333

[7] J. Lazarou, B.H. Pomeranz, Corey P.N, JAMA.279 (1998)1200-5

[8] A.K. Daly Curr Opin Drug Discov Devel.10 (2007) 29-36

[9] http://www.amplichip.us/

[10] Jiunn H. Lin, et al., Current Drug Metabolism 8 (2007) 109-136

[11] S. Carrara, et al., proceedings of the IEEE/ICME 2009

[12] David Ogle, Journal of Chromatography A, 979 (2002) 155-161

[13] Danielson P Curr Drug Metab 3 (2002) 561-97

[14] S. Carrara, et al., Biosensors and Bioelectronics, 24(2008) 148150

[15] Kang, S. J., et al., Nature Nanotechnology, 2007, pp. 230-236.

[16] Pengfei Qi, et al., Nano Letters, 2003, Vol.3 347-351.

[17] Keith Bradley., et al., Nano Letters, 2005, Vol.5 841-845.

[18] Johnson, D.L., et al., Biochemical Pharmacology 69 (2005)

1533-1541

[19] G Gruner, "Carbon nanotube transistors for biosensing applications", Analytical and bioanalytical chemistry, 2006 Springer.

[20] Zhang, J., et al., IEEE Proc. DATE, pp. 1009 - 1014, 2008.

[21] Wong H.-S. P., et al., " Proc. Intl. Solid State Circuits Conf., pp. $370-371,2003$. 